\title{
Nachhaltigkeit und Linguistik. Sprachwissenschaftliche Innovationen im Kontext einer globalen Thematik
}

Nachhaltigkeit und nachhaltige Entwicklung gehören zu den drängenden globalen Zielen unserer Zeit. Als interdisziplinäres und vielschichtiges Thema ist Nachhaltigkeit auch für die angewandte Linguistik hochrelevant - sei es mit Blick auf die diskursive Debattenkultur, neue mediale Formen der Partizipation oder Formen der Wissenskommunikation, wie die international entstandene Nachhaltigkeitskommunikation in Wirtschaft und Politik.

Als interdisziplinäres sowie sprach- und kommunikationsraumübergreifendes Thema erfordert eine linguistische Beschäftigung mit Nachhaltigkeit differenzierte Blickwinkel auf eine Vielfalt von Formen und Kommunikationssituationen. Daher setzt dieses Heft über den thematischen Fokus Nachhaltigkeit hinaus einen dezidiert methodischen Schwerpunkt. Es werden verschiedene innovative linguistische Methoden (teilweise neue, teilweise bewährte Verfahren in neuer Anwendung und Kombination) verwendet und diskutiert, um sich dem internationalen und komplexen Phänomen Nachhaltigkeit zu nähern: Zum einen werden verschiedene diskursive Nachhaltigkeitsdebatten und (wirtschafts-) politische Vermittlungskommunikate transnational und sprachvergleichend, argumentationsanalytisch, kritisch-diskursanalytisch, multimodal oder sprachkritisch untersucht. Damit können kommunikative Praktiken beleuchtet werden, die im Rahmen des vielschichtigen Themas Nachhaltigkeit relevant sind. Zum anderen wird aufgezeigt, dass die Linguistik als angewandte oder gar transformative Wissenschaft einen Beitrag zu nachhaltiger Entwicklung und zu Aushandlungen um Nachhaltigkeit leisten kann.

Der Aufsatz von Anna Mattfeldt zeigt am Beispiel des Frackingdiskurses auf, wie eine sprach- und kulturvergleichende Diskursanalyse zu einer international debattierten Herausforderung durchgeführt werden kann, und nimmt dabei agonale Aushandlungen in den Blick. Filtereffekte, die durch regional und national geprägte Perspektiven auf das Thema entstehen, werden gerade in der kontrastiven Betrachtung besonders sichtbar herausgearbeitet. Thomas Metten legt im zweiten Text des Heftes dar, dass die Gestaltung von Prozessen der wechselseitigen Teilhabe von Wissenschaft und Gesellschaft als zentrale Aufgabe einer Wissenschaftskommunikation gelten sollte, und plädiert für eine Beteiligung der Linguistik an transdisziplinären Vorhaben, um konkretes Handlungswissen für eine gesellschaftliche Transformation hin zu einer nachhaltigen Entwicklung mit zu generieren. Natürlich ist dieses Ziel der Nachhaltigkeit normativ und wird nicht von allen Menschen, politischen Entscheider/-innen usw. geteilt. Selbst wenn ein solcher Konsens vorhanden ist, eine nachhaltige Entwicklung anzustreben, kann auch hier aufgedeckt werden, dass Konflikte latent innerhalb zentraler argumentativer Begriffe liegen insbesondere hinsichtlich der Frage, wie der Weg zur Nachhaltigkeit begangen werden sollte. Dieses komplexe tiefensemantische Konfliktpotenzial entwirrt Carolin Schwegler beispielhaft mittels eines framesemantischen Ansatzes für Argumentationen mit Gerechtigkeit.

Der Tatsache, dass die Debatte um Nachhaltigkeit zunehmend auch multimodal geführt wird, trägt der Zugang von Felix Böhm und Paul Reszke Rechnung, der damit eine weitere Dimension zum linguistischen Analysespektrum des Nachhaltigkeitsdiskurses hinzufügt. 
Ebenso zeigt der Aufsatz, dass Performances (als multimodale Handlungen) den Themenkomplex Nachhaltigkeit nicht nur verhandeln, sondern auch explizit zu Handlungsveränderungen der Rezipierenden aufrufen. Martin Reisigls Text nimmt darauffolgend - ganz im Sinne der Critical Discourse Analysis - eine direkte und spezifische Positionierung zum Nachhaltigkeitsthema individuelle Mobilität vor. Kritisch-wertende linguistische Beiträge wie dieser können an der o. g. transdisziplinären Schnittstelle eine wichtige Rolle spielen, wenn man sie als empirisch informierte Wertung und als Diskursbeitrag versteht. Ruth M. Mell verdeutlicht anschließend, dass die Linguistik auf mehreren wissenskommunikativ relevanten Ebenen Möglichkeiten zur Beteiligung an der Nachhaltigkeitsdebatte bieten kann. Sie zeigt exemplarisch an Sprachgebrauchsmustern bioethischer Terminologie den Mehrwert einer quantitativen Analyse nachhaltigkeitsbezogener Aushandlungen auf.

Wir hoffen, dass die Beiträge dieses Themenhefts zum einen das Potenzial der Linguistik für die Nachhaltigkeitsdebatte und die nachhaltige Entwicklung aufzeigen, zum anderen die Möglichkeiten des Themas Nachhaltigkeit für die linguistische Forschung verdeutlichen. Abschließend möchten wir den anonymen externen Gutachter/-innen der Aufsätze sowie den Gutachter/-innen des Herausgeberbeirats der Zeitschrift Deutsche Sprache herzlich für ihre wertvollen Hinweise und konstruktiven Kommentare danken. Wir danken außerdem Melanie Kraus und Annett Patzschewitz für die freundliche redaktionelle Betreuung sowie das Lektorat und die sorgfältige Einrichtung der Druckvorlage. 